\title{
Solution and Hyers-Ulam-Rassias Stability of Generalized Mixed Type Additive-Quadratic Functional Equations in Fuzzy Banach Spaces
}

\author{
M. Eshaghi Gordji, ${ }^{1}$ H. Azadi Kenary, ${ }^{2}$ H. Rezaei, ${ }^{2}$ \\ Y. W. Lee, ${ }^{3}$ and G. H. Kim ${ }^{4}$ \\ ${ }^{1}$ Department of Mathematics, Semnan University, Semnan 35131-19111, Iran \\ ${ }^{2}$ Department of Mathematics, Yasouj University, Yasouj 75918-74831, Iran \\ ${ }^{3}$ Department of Computer Hacking and Information Security, Daejeon University, \\ Youngwoondong Donggu, Daejeon 300-716, Republic of Korea \\ ${ }^{4}$ Department of Mathematics, Kangnam University, Yongin, Gyeonggi 446-702, Republic of Korea
}

Correspondence should be addressed to G. H. Kim, ghkim@kangnam.ac.kr

Received 19 November 2011; Revised 21 January 2012; Accepted 13 February 2012

Academic Editor: Gerd Teschke

Copyright (C) 2012 M. Eshaghi Gordji et al. This is an open access article distributed under the Creative Commons Attribution License, which permits unrestricted use, distribution, and reproduction in any medium, provided the original work is properly cited.

By using fixed point methods and direct method, we establish the generalized Hyers-Ulam stability of the following additive-quadratic functional equation $f(x+k y)+f(x-k y)=f(x+$ $y)+f(x-y)+(2(k+1) / k) f(k y)-2(k+1) f(y)$ for fixed integers $k$ with $k \neq 0, \pm 1$ in fuzzy Banach spaces.

\section{Introduction and Preliminaries}

The stability problem of functional equations was originated from a question of Ulam [1] in 1940, concerning the stability of group homomorphisms. Let $\left(G_{1}, \cdot\right)$ be a group and let $\left(G_{2}, *, d\right)$ be a metric group with the metric $d(\cdot, \cdot)$. Given $\epsilon>0$, does there exist a $\delta>0$, such that if a mapping $h: G_{1} \rightarrow G_{2}$ satisfies the inequality $d(h(x \cdot y), h(x) * h(y))<\delta$ for all $x, y \in G_{1}$, then there exists a homomorphism $H: G_{1} \rightarrow G_{2}$ with $d(h(x), H(x))<\epsilon$ for all $x \in G_{1}$ ? In other words, under what condition does there exist a homomorphism near an approximate homomorphism? The concept of stability for functional equation arises when we replace the functional equation by an inequality which acts as a perturbation of the equation. In 1941, Hyers [2] gave a first affirmative answer to the question of Ulam for Banach spaces. Let $f: E \rightarrow E^{\prime}$ be a mapping between Banach spaces such that

$$
\|f(x+y)-f(x)-f(y)\| \leq \delta,
$$


for all $x, y \in E$, and for some $\delta>0$. Then there exists a unique additive mapping $T: E \rightarrow E^{\prime}$ such that

$$
\|f(x)-T(x)\| \leq \delta
$$

for all $x \in E$. Moreover if $f(t x)$ is continuous in $t \in \mathbb{R}$ for each fixed $x \in E$, then $T$ is linear. In 1978, Rassias [3] provided a generalization of Hyers' Theorem which allows the Cauchy difference to be unbounded. In 1991, Gajda [4] answered the question for the case $p>1$, which was raised by Rassias. This new concept is known as Hyers-Ulam-Rassias stability of functional equations (see [5-17]).

The functional equation

$$
f(x+y)+f(x-y)=2 f(x)+2 f(y)
$$

is related to a symmetric biadditive function. It is natural that this equation is called a quadratic functional equation. In particular, every solution of the quadratic equation (1.3) is said to be a quadratic function. It is well known that a function $f$ between real vector spaces is quadratic if and only if there exists a unique symmetric biadditive function $B$ such that $f(x)=B(x, x)$ for all $x$ (see $[6,18])$. The biadditive function $B$ is given by

$$
B(x, y)=\frac{1}{4}(f(x+y)-f(x-y)) .
$$

A Hyers-Ulam-Rassias stability problem for the quadratic functional equation (1.3) was proved by Skof for functions $f: A \rightarrow B$, where $A$ is normed space and $B$ Banach space (see [19-22]). Borelli and Forti [23] generalized the stability result of quadratic functional equations as follows (cf. [24, 25]): let $G$ be an Abelian group, and $X$ a Banach space. Assume that a mapping $f: G \rightarrow X$ satisfies the functional inequality:

$$
\|f(x+y)+f(x-y)-2 f(x)-2 f(y)\| \leq \varphi(x, y),
$$

for all $x, y \in G$, and $\varphi: G \times G \rightarrow[0, \infty)$ is a function such that

$$
\Phi(x, y):=\sum_{i=0}^{\infty} \frac{1}{4^{i+1}} \varphi\left(2^{i} x, 2^{i} y\right)<\infty
$$

for all $x, y \in G$. Then there exists a unique quadratic mapping $Q: G \rightarrow X$ with the property

$$
\|f(x)-Q(x)\| \leq \Phi(x, x)
$$

for all $x \in G$.

Now, we introduce the following functional equation for fixed integers $k$ with $k \neq 0, \pm 1$ :

$$
f(x+k y)+f(x-k y)=f(x+y)+f(x-y)+\frac{2(k+1)}{k} f(k y)-2(k+1) f(y)
$$


with $f(0)=0$ in a non-Archimedean space. It is easy to see that the function $f(x)=a x+b x^{2}$ is a solution of the functional equation (1.8), which explains why it is called additive-quadratic functional equation. For more detailed definitions of mixed type functional equations, we can refer to [26-47].

Definition 1.1 (see [48]). Let $X$ be a real vector space. A function $N: X \times \mathbb{R} \rightarrow[0,1]$ is called a fuzzy norm on $X$ if for all $x, y \in X$ and all $s, t \in \mathbb{R}$,

(N1) $N(x, t)=0$ for $t \leq 0$;

(N2) $x=0$ if and only if $N(x, t)=1$ for all $t>0$;

(N3) $N(c x, t)=N(x, t /|c|)$ if $c \neq 0$;

(N4) $N(x+y, s+t) \geq \min \{N(x, s), N(y, t)\}$;

(N5) $N(x, \cdot)$ is a nondecreasing function of $\mathbb{R}$ and $\lim _{t \rightarrow \infty} N(x, t)=1$;

(N6) for $x \neq 0, N(x, \cdot)$ is continuous on $\mathbb{R}$.

The pair $(X, N)$ is called a fuzzy normed vector space.

Example 1.2. Let $(X,\|\cdot\|)$ be a normed linear space and $\alpha, \beta>0$. Then

$$
N(x, t)= \begin{cases}\frac{\alpha t}{\alpha t+\beta\|x\|}, & t>0, x \in X, \\ 0, & t \leq 0, x \in X\end{cases}
$$

is a fuzzy norm on $X$.

Definition 1.3. Let $(X, N)$ be a fuzzy normed vector space. A sequence $\left\{x_{n}\right\}$ in $X$ is said to be convergent or converge if there exists an $x \in X$ such that $\lim _{n \rightarrow \infty} N\left(x_{n}-x, t\right)=1$ for all $t>0$. In this case, $x$ is called the limit of the sequence $\left\{x_{n}\right\}$ in $X$ and one denotes it by $N-\lim _{n \rightarrow \infty} x_{n}=x$.

Definition 1.4. Let $(X, N)$ be a fuzzy normed vector space. A sequence $\left\{x_{n}\right\}$ in $X$ is called Cauchy if for each $\epsilon>0$ and each $t>0$ there exists an $n_{0} \in \mathbb{N}$ such that for all $n \geq n_{0}$ and all $p>0$, one has $N\left(x_{n+p}-x_{n}, t\right)>1-\epsilon$.

It is well known that every convergent sequence in a fuzzy normed vector space is Cauchy. If each Cauchy sequence is convergent, then the fuzzy norm is said to be complete and the fuzzy normed vector space is called a fuzzy Banach space.

Example 1.5. Let $N: \mathbb{R} \times \mathbb{R} \rightarrow[0,1]$ be a fuzzy norm on $\mathbb{R}$ defined by

$$
N(x, t)= \begin{cases}\frac{t}{t+|x|}, & t>0, \\ 0, & t \leq 0 .\end{cases}
$$


The $(\mathbb{R}, N)$ is a fuzzy Banach space. Let $\left\{x_{n}\right\}$ be a Cauchy sequence in $\mathbb{R}, \delta>0$, and $\epsilon=$ $\delta /(1+\delta)$. Then there exist $m \in \mathbb{N}$ such that for all $n \geq m$ and all $p>0$, one has

$$
\frac{1}{1+\left|x_{n+p}-x_{n}\right|} \geq 1-\epsilon
$$

So $\left|x_{n+p}-x_{n}\right|<\delta$ for all $n \geq m$ and all $p>0$. Therefore $\left\{x_{n}\right\}$ is a Cauchy sequence in $(\mathbb{R},|\cdot|)$. Let $x_{n} \rightarrow x_{0} \in \mathbb{R}$ as $n \rightarrow \infty$. Then $\lim _{n \rightarrow \infty} N\left(x_{n}-x_{0}, t\right)=1$ for all $t>0$.

We say that a mapping $f: X \rightarrow Y$ between fuzzy normed vector spaces $X$ and $Y$ is continuous at a point $x \in X$ if for each sequence $\left\{x_{n}\right\}$ converging to $x_{0} \in X$, the sequence $\left\{f\left(x_{n}\right)\right\}$ converges to $f\left(x_{0}\right)$. If $f: X \rightarrow Y$ is continuous at each $x \in X$, then $f: X \rightarrow Y$ is said to be continuous on $X([49])$.

Definition 1.6. Let $X$ be a set. A function $d: X \times X \rightarrow[0, \infty]$ is called a generalized metric on $X$ if $d$ satisfies the following conditions:

(1) $d(x, y)=0$ if and only if $x=y$ for all $x, y \in X$;

(2) $d(x, y)=d(y, x)$ for all $x, y \in X$;

(3) $d(x, z) \leq d(x, y)+d(y, z)$ for all $x, y, z \in X$.

Theorem 1.7. Let $(X, d)$ be a complete generalized metric space and let $J: X \rightarrow X$ be a strictly contractive mapping with Lipschitz constant $L<1$. Then, for all $x \in X$, either

$$
d\left(J^{n} x, J^{n+1} x\right)=\infty
$$

for all nonnegative integers $n$, or there exists a positive integer $n_{0}$ such that

(1) $d\left(J^{n} x, J^{n+1} x\right)<\infty$ for all $n_{0} \geq n_{0}$;

(2) the sequence $\left\{J^{n} x\right\}$ converges to a fixed point $y^{*}$ of $J$;

(3) $y^{*}$ is the unique fixed point of $J$ in the set $Y=\left\{y \in X: d\left(J^{n_{0}} x, y\right)<\infty\right\}$;

(4) $d\left(y, y^{*}\right) \leq 1 /(1-L) d(y, J y)$ for all $y \in Y$.

We have the following theorem from [42], which investigates the solution of (1.8).

Theorem 1.8. A function $f: X \rightarrow Y$ with $f(0)=0$ satisfies (1.8) for all $x, y \in X$ if and only if there exist functions $A: X \rightarrow Y$ and $Q: X \times X \rightarrow Y$, such that $f(x)=A(x)+Q(x, x)$ for all $x \in X$, where the function $Q$ is symmetric biadditive and $A$ is additive.

\section{A Fixed Point Method}

Using the fixed point methods, we prove the Hyers-Ulam stability of the additive-quadratic functional equation (1.8) in fuzzy Banach spaces. Throughout this paper, assume that $X$ is a vector space and that $(Y, N)$ is a fuzzy Banach space. 
Theorem 2.1. Let $\varphi: X^{2} \rightarrow[0, \infty)$ be a mapping such that there exists an $\alpha<1$ with

$$
\varphi(x, y) \leq|k| \alpha \varphi\left(\frac{x}{k}, \frac{y}{k}\right)
$$

for all $x, y \in X$. Let $f: X \rightarrow Y$ be an odd function satisfying $f(0)=0$ and

$$
\begin{aligned}
& N\left(f(k(x+y))+f(k(x-y))-f(k x+y)-f(k x-y)-\frac{2(k+1)}{k} f(k y)+2(k+1) f(y), t\right) \\
& \quad \geq \frac{t}{t+\varphi(x, y)},
\end{aligned}
$$

for all $x, y \in X$ and all $t>0$. Then $A(x):=N-\lim _{n \rightarrow \infty}\left(f\left(k^{n} x\right) / k^{n}\right)$ exists for all $x \in X$ and defines a unique additive mapping $A: X \rightarrow Y$ such that

$$
N(f(x)-A(x), t) \geq \frac{(|2 k+2|-|2 k+2| \alpha) t}{(|2 k+2|-|2 k+2| \alpha) t+\varphi(0, x)}
$$

for all $x \in X$ and $t>0$.

Proof. Note that $f(0)=0$ and $f(-x)=-f(x)$ for all $x \in X$ since $f$ is an odd function. Putting $x=0$ in (2.2), we get

$$
N\left(\frac{f(k y)}{k}-f(y), \frac{t}{|2 k+2|}\right) \geq \frac{t}{t+\varphi(0, y)}
$$

for all $y \in X$ and all $t>0$. Replacing $y$ by $x$ in (2.4), we have

$$
N\left(\frac{f(k x)}{k}-f(x), \frac{t}{|2 k+2|}\right) \geq \frac{t}{t+\varphi(0, x)}
$$

for all $x \in X$ and all $t>0$. Consider the set $S:=\{h: X \rightarrow Y ; h(0)=0\}$ and introduce the generalized metric on $S$ :

$$
d(g, h)=\inf _{\mu \in(0,+\infty)}\left\{N(g(x)-h(x), \mu t) \geq \frac{t}{t+\varphi(0, x)}, \forall x \in X\right\}
$$

where, as usual, $\inf \phi=+\infty$. It is easy to show that $(S, d)$ is complete (see [50]). We consider the mapping $J:(S, d) \rightarrow(S, d)$ as follows:

$$
J g(x):=\frac{1}{k} g(k x)
$$


for all $x \in X$. Let $g, h \in S$ be given such that $d(g, h)=\beta$. Then

$$
N(g(x)-h(x), \beta t) \geq \frac{t}{t+\varphi(0, x)}
$$

for all $x \in X$ and all $t>0$. Hence

$$
\begin{aligned}
N(J g(x)-J h(x), \alpha \beta t) & =N\left(\frac{1}{k} g(k x)-\frac{1}{k} h(k x), \alpha \beta t\right) \\
& =N(g(k x)-h(k x),|k| \alpha \beta t) \\
& \geq \frac{|k| \alpha t}{|k| \alpha t+\varphi(0, x)} \\
& \geq \frac{|k| \alpha t}{|k| \alpha t+|k| \alpha \varphi(0, x)} \\
& =\frac{t}{t+\varphi(0, x)}
\end{aligned}
$$

for all $x \in X$ and all $t>0$. So $d(g, h)=\beta$ implies that $d(J g, J h) \leq \alpha \beta$. This means that $d(J g, J h) \leq \alpha d(g, h)$ for all $g, h \in S$. It follows from (2.5) that

$$
d(f, J f) \leq \frac{1}{|2 k+2|}
$$

By Theorem 1.7, there exists a mapping $A: X \rightarrow Y$ satisfying the following.

(1) $A$ is a fixed point of $J$, that is,

$$
k A(x)=A(k x)
$$

for all $x \in X$. The mapping $A$ is a unique fixed point of $J$ in the set $M=\{g \in S: d(h, g)<\infty\}$. This implies that $A$ is a unique mapping satisfying (2.11) such that there exists a $\mu \in(0, \infty)$ satisfying

$$
N(f(x)-A(x), \mu t) \geq \frac{t}{t+\varphi(0, x)}
$$

for all $x \in X$.

(2) $d\left(J^{n} f, A\right) \rightarrow 0$ as $n \rightarrow \infty$. This implies the equality $\lim _{n \rightarrow \infty}\left(f\left(k^{n} x\right) / k^{n}\right)=A(x)$, for all $x \in X$.

(3) $d(f, A) \leq(1 /(1-\alpha)) d(f, J f)$, which implies the inequality

$$
d(f, A) \leq \frac{1}{|2 k+2|-|2 k+2| \alpha} .
$$

This implies that the inequality (2.3) holds. 
It follows from (2.1) and (2.2) that

$$
\begin{aligned}
& N\left(\frac{f\left(k^{n}(x+k y)\right)}{k^{n}}+\frac{f\left(k^{n}(x-k y)\right)}{k^{n}}-\frac{f\left(k^{n}(x+y)\right)}{k^{n}}-\frac{f\left(k^{n}(x-y)\right)}{k^{n}}\right. \\
& \left.\quad-\frac{2(k+1)}{k} \frac{f\left(k^{n+1} y\right)}{k^{n}}+2(k+1) \frac{f\left(k^{n} y\right)}{k^{n}}, \frac{t}{k^{n}}\right) \\
& \quad \geq \frac{t}{t+\varphi\left(k^{n} x, k^{n} y\right)},
\end{aligned}
$$

for all $x, y \in X$, all $t>0$, and all $n \in \mathbb{N}$. So

$$
\begin{aligned}
& N\left(\frac{f\left(k^{n}(x+k y)\right)}{k^{n}}+\frac{f\left(k^{n}(x-k y)\right)}{k^{n}}-\frac{f\left(k^{n}(x+y)\right)}{k^{n}}-\frac{f\left(k^{n}(x-y)\right)}{k^{n}}\right. \\
& \left.\quad-\frac{2(k+1)}{k} \frac{f\left(k^{n+1} y\right)}{k^{n}}+2(k+1) \frac{f\left(k^{n} y\right)}{k^{n}}, t\right) \\
& \quad \geq \frac{|k|^{n} t}{|k|^{n} t+|k|^{n} \alpha^{n} \varphi(x, y)},
\end{aligned}
$$

for all $x, y \in X$, all $t>0$, and all $n \in \mathbb{N}$. Since $\lim _{n \rightarrow \infty}\left(|k|^{n} t /\left(|k|^{n} t+|k|^{n} \alpha^{n} \varphi(x, y)\right)\right)=1$ for all $x, y \in X$ and all $t>0$, we obtain that

$$
\begin{aligned}
& N\left(A(k(x+y))+A(k(x-y))-A(k x+y)-A(k x-y)-\frac{2(k+1)}{k} A(k y)\right. \\
& \quad+2(k+1) A(y), t)=1
\end{aligned}
$$

for all $x, y, z \in X$ and all $t>0$. Hence the mapping $A: X \rightarrow Y$ is additive, as desired.

Corollary 2.2. Let $\theta \geq 0$ and let $r$ be a real positive number with $r<1$. Let $X$ be a normed vector space with norm $\|\cdot\|$. Let $f: X \rightarrow Y$ be an odd mapping satisfying

$$
\begin{aligned}
& N\left(f(k(x+y))+f(k(x-y))-f(k x+y)-f(k x-y)-\frac{2(k+1)}{k} f(k y)+2(k+1) f(y), t\right) \\
& \quad \geq \frac{t}{t+\theta\left(\|x\|^{r}+\|y\|^{r}\right)},
\end{aligned}
$$

for all $x, y \in X$ and all $t>0$. Then the limit $A(x):=N-\lim _{n \rightarrow \infty}\left(f\left(k^{n} x\right) / k^{n}\right)$ exists for each $x \in X$ and defines a unique additive mapping $A: X \rightarrow Y$ such that

$$
N(f(x)-A(x), t) \geq \frac{|2 k+2|\left(|k|-|k|^{r}\right) t}{|2 k+2|\left(|k|-|k|^{r}\right) t+\left.|k| \theta|| x\right|^{r}},
$$

for all $x \in X$ and all $t>0$. 
Proof. The proof follows from Theorem 2.1 by taking $\varphi(x, y):=\theta\left(\|x\|^{r}+\|y\|^{r}\right)$ for all $x, y \in X$. Then we can choose $\alpha=|k|^{r-1}$ and we get the desired result.

Theorem 2.3. Let $\varphi: X^{2} \rightarrow[0, \infty)$ be a mapping such that there exists an $\alpha<1$ with

$$
\varphi\left(\frac{x}{k}, \frac{y}{k}\right) \leq \frac{\alpha}{|k|} \varphi(x, y)
$$

for all $x, y \in X$. Let $f: X \rightarrow Y$ be an odd mapping satisfying $f(0)=0$ and (2.2). Then the limit $A(x):=N-\lim _{n \rightarrow \infty} k^{n} f\left(x / k^{n}\right)$ exists for all $x \in X$ and defines a unique additive mapping $A: X \rightarrow Y$ such that

$$
N(f(x)-A(x), t) \geq \frac{(|2 k+2|-|2 k+2| \alpha) t}{(|2 k+2|-|2 k+2| \alpha) t+\alpha \varphi(0, x)}
$$

for all $x \in X$ and all $t>0$.

Proof. Let $(S, d)$ be the generalized metric space defined as in the proof of Theorem 2.1.

Consider the mapping $J: S \rightarrow S$ by

$$
J g(x):=k g\left(\frac{x}{k}\right)
$$

for all $g \in S$. Let $g, h \in S$ be given such that $d(g, h)=\beta$. Then

$$
N(g(x)-h(x), \beta t) \geq \frac{t}{t+\varphi(0, x)}
$$

for all $x \in X$ and all $t>0$. Hence

$$
\begin{aligned}
N(J g(x)-J h(x), \alpha \beta t) & =N\left(k g\left(\frac{x}{k}\right)-k h\left(\frac{x}{k}\right), \alpha \beta t\right) \\
& =N\left(g\left(\frac{x}{k}\right)-h\left(\frac{x}{k}\right), \frac{\alpha \beta t}{|k|}\right) \\
& \geq \frac{(\alpha t /|k|)}{\alpha t /|k|+\varphi(0, x / k)} \geq \frac{t}{t+\varphi(0, x)},
\end{aligned}
$$

for all $x \in X$ and all $t>0$. So $d(g, h)=\beta$ implies that $d(J g, J h) \leq \alpha \beta$. This means that $d(J g, J h) \leq \alpha d(g, h)$ for all $g, h \in S$. It follows from (2.5) that

$$
N\left(k f\left(\frac{x}{k}\right)-f(x), \frac{k t}{|2 k+2|}\right) \geq \frac{t}{t+\varphi(0, x / k)} \geq \frac{t}{t+(\alpha /|k|) \varphi(0, x)}
$$


for all $x \in X$ and all $t>0$. Therefore

$$
N\left(k f\left(\frac{x}{k}\right)-f(x), \frac{\alpha t}{|2 k+2|}\right) \geq \frac{t}{t+\varphi(0, x)} .
$$

So $d(f, J f) \leq \alpha$. By Theorem 1.7 , there exists a mapping $A: X \rightarrow Y$ satisfying the following. (1) $A$ is a fixed point of $J$, that is,

$$
A\left(\frac{x}{k}\right)=\frac{1}{k} A(x)
$$

for all $x \in X$. The mapping $A$ is a unique fixed point of $J$ in the set $\Omega=\{h \in S: d(g, h)<\infty\}$. This implies that $A$ is a unique mapping satisfying (2.26) such that there exists $\mu \in(0, \infty)$ satisfying

$$
N(f(x)-A(x), \mu t) \geq \frac{t}{t+\varphi(0, x)}
$$

for all $x \in X$ and $t>0$.

(2) $d\left(J^{n} f, A\right) \rightarrow 0$ as $n \rightarrow \infty$. This implies the equality $N-\lim _{n \rightarrow \infty} k^{n} f\left(x / k^{n}\right)=A(x)$ for all $x \in X$.

(3) $d(f, A) \leq d(f, J f) /(1-L)$ with $f \in \Omega$, which implies the inequality

$$
d(f, A) \leq \frac{\alpha}{|2 k+2|-|2 k+2| \alpha} .
$$

This implies that the inequality (2.20) holds.

The rest of proof is similar to the proof of Theorem 2.1.

Corollary 2.4. Let $\theta \geq 0$ and let $r$ be a real number with $r>1$. Let $X$ be a normed vector space with norm $\|\cdot\|$. Let $f: X \rightarrow Y$ be an odd mapping satisfying (2.17). Then $A(x):=$ $N-\lim _{n \rightarrow \infty} k^{n} f\left(x / k^{n}\right)$ exists for each $x \in X$ and defines a unique additive mapping $A: X \rightarrow Y$ such that

$$
N(f(x)-A(x), t) \geq \frac{|2 k+2|\left(|k|^{r}-|k|\right) t}{|2 k+2|\left(|k|^{r}-|k|\right) t+\left.|k| \theta|| x\right|^{r}}
$$

for all $x \in X$ and all $t>0$.

Proof. The proof follows from Theorem 2.3 by taking $\varphi(x, y):=\theta\left(\|x\|^{r}+\|y\|^{r}\right)$ for all $x, y \in X$. Then we can choose $\alpha=|k|^{1-r}$ and we get the desired result.

Theorem 2.5. Let $\varphi: X^{2} \rightarrow[0, \infty)$ be a function such that there exists an $\alpha<1$ with

$$
\varphi(x, y) \leq k^{2} \alpha \varphi\left(\frac{x}{k}, \frac{y}{k}\right)
$$


for all $x, y \in X$. Let $f: X \rightarrow Y$ be an even mapping with $f(0)=0$ and satisfying (2.2). Then $Q(x):=N-\lim _{n \rightarrow \infty}\left(f\left(k^{n} x\right) / k^{2 n}\right)$ exists for all $x \in X$ and defines a unique quadratic mapping $Q: X \rightarrow Y$ such that

$$
N(f(x)-Q(x), t) \geq \frac{(2|k|-2|k| \alpha) t}{(2|k|-2|k| \alpha) t+\varphi(0, x)}
$$

for all $x \in X$ and all $t>0$.

Proof. Replacing $x$ by $k x$ in (2.2), we get

$$
\begin{aligned}
& N\left(f(k(x+y))+f(k(x-y))-f(k x+y)-f(k x-y)-\frac{2(k+1)}{k} f(k y)+2(k+1) f(y), t\right) \\
& \quad \geq \frac{t}{t+\varphi(k x, y)},
\end{aligned}
$$

for all $x, y \in X$ and all $t>0$. Putting $x=0$ and replacing $y$ by $x$ in (2.32), we have

$$
N\left(\frac{f(k x)}{k}-k f(x), \frac{t}{2}\right) \geq \frac{t}{t+\varphi(0, x)}
$$

for all $x \in X$ and all $t>0$. By (2.33), (N3), and (N4), we get

$$
N\left(\frac{f(k x)}{k^{2}}-f(x), \frac{t}{2|k|}\right) \geq \frac{t}{t+\varphi(0, x)}
$$

for all $x \in X$ and all $t>0$. Consider the set $S^{*}:=\{h: X \rightarrow Y ; h(0)=0\}$ and introduce the generalized metric on $S^{*}$ :

$$
d(g, h)=\inf _{\mu \in(0,+\infty)}\left\{N(g(x)-h(x), \mu t) \geq \frac{t}{t+\varphi(0, x)}, \forall x \in X\right\}
$$

where, as usual, $\inf \phi=+\infty$. It is easy to show that $\left(S^{*}, d\right)$ is complete (see [50]). Now we consider the linear mapping $J:\left(S^{*}, d\right) \rightarrow\left(S^{*}, d\right)$ such that

$$
J g(x):=\frac{1}{k^{2}} g(k x)
$$

for all $x \in X$. Proceeding as in the proof of Theorem 2.1, we obtain that $d(g, h)=\beta$ implies that $d(J g, J h) \leq \alpha \beta$. This means that $d(J g, J h) \leq \alpha d(g, h)$ for all $g, h \in S$. It follows from 
(2.34) that

$$
d(f, J f) \leq \frac{1}{2|k|}
$$

By Theorem 1.7, there exists a mapping $Q: X \rightarrow Y$ such that one has the folowing.

(1) $Q$ is a fixed point of $J$, that is,

$$
k^{2} Q(x)=Q(k x)
$$

for all $x \in X$. The mapping $Q$ is a unique fixed point of $J$ in the set $M=\left\{g \in S^{*}: d(h, g)<\infty\right\}$. This implies that $Q$ is a unique mapping satisfying (2.38) such that there exists a $\mu \in(0, \infty)$ satisfying $N(f(x)-Q(x), \mu t) \geq t /(t+\varphi(0, x))$ for all $x \in X$.

(2) $d\left(J^{n} f, Q\right) \rightarrow 0$ as $n \rightarrow \infty$. This implies the equality $\lim _{n \rightarrow \infty}\left(f\left(k^{n} x\right) / k^{2 n}\right)=Q(x)$ for all $x \in X$.

(3) $d(f, Q) \leq(1 /(1-\alpha)) d(f, J f)$, which implies the inequality $d(f, Q) \leq 1 /(2|k|-$ $2|k| \alpha)$. This implies that the inequality (2.31) holds.

The rest of the proof is similar to the proof of Theorem 2.1.

Corollary 2.6. Let $\theta \geq 0$ and let $r$ be a real positive number with $r<1$. Let $X$ be a normed vector space with norm $\|\cdot\|$. Let $f: X \rightarrow Y$ be an even mapping with $f(0)=0$ and satisfying (2.17). Then the limit $Q(x):=N-\lim _{n \rightarrow \infty}\left(f\left(k^{n} x\right) / k^{2 n}\right)$ exists for each $x \in X$ and defines a unique quadratic mapping $Q: X \rightarrow Y$ such that

$$
N(f(x)-Q(x), t) \geq \frac{\left(2 k^{2}-2 k^{2 r}\right) t}{\left(2 k^{2}-2 k^{2 r}\right) t+|k| \theta\|x\|^{r}}
$$

for all $x \in X$ and all $t>0$.

Proof. The proof follows from Theorem 2.5 by taking $\varphi(x, y):=\theta\left(\|x\|^{r}+\|y\|^{r}\right)$ for all $x, y \in X$. Then we can choose $\alpha=k^{2 r-2}$ and we get the desired result.

Theorem 2.7. Let $\varphi: X^{2} \rightarrow[0, \infty)$ be a function such that there exists an $\alpha<1$ with

$$
\varphi\left(\frac{x}{k}, \frac{y}{k}\right) \leq \frac{\alpha}{k^{2}} \varphi(x, y)
$$

for all $x, y \in X$. Let $f: X \rightarrow Y$ be an even mapping with $f(0)=0$ and satisfying (2.2). Then the limit $Q(x):=N-\lim _{n \rightarrow \infty} k^{2 n} f\left(x / k^{n}\right)$ exists for all $x \in X$ and defines a unique quadratic mapping $Q: X \rightarrow Y$ such that

$$
N(f(x)-Q(x), t) \geq \frac{(2|k|-2|k| \alpha) t}{(2|k|-2|k| \alpha) t+\alpha \varphi(0, x)}
$$

for all $x \in X$ and $t>0$. 
Proof. Let $\left(S^{*}, d\right)$ be the generalized metric space defined as in the proof of Theorem 2.5. It follows from (2.34) that

$$
N\left(k^{2} f\left(\frac{x}{k}\right)-f(x), \frac{|k| t}{2}\right) \geq \frac{t}{t+\varphi(0, x / k)} \geq \frac{t}{t+\left(\alpha / k^{2}\right) \varphi(0, x)},
$$

for all $x \in X$ and $t>0$. So

$$
N\left(f(x)-k^{2} f\left(\frac{x}{k}\right), \frac{\alpha t}{2|k|}\right) \geq \frac{t}{t+\varphi(0, x)} .
$$

The rest of the proof is similar to the proofs of Theorems 2.1 and 2.3.

Corollary 2.8. Let $\theta \geq 0$ and let $r$ be a real number with $r>1$. Let $X$ be a normed vector space with norm $\|\cdot\|$. Let $f: X \rightarrow Y$ be an even mapping with $f(0)=0$ and satisfying (2.17). Then $Q(x):=N-\lim _{n \rightarrow \infty} k^{2 n} f\left(x / k^{n}\right)$ exists for each $x \in X$ and defines a unique quadratic mapping $Q: X \rightarrow Y$ such that

$$
N(f(x)-Q(x), t) \geq \frac{\left(2|k|^{2 r+1}-2|k|^{3}\right) t}{\left(2|k|^{2 r+1}-2|k|^{3}\right) t+k^{2} \theta\|x\|^{r}}
$$

for all $x \in X$ and all $t>0$.

Proof. It follows from Theorem 2.7 by taking $\varphi(x, y):=\theta\left(\|x\|^{r}+\|y\|^{r}\right)$ for all $x, y \in X$. Then we can choose $\alpha=k^{2-2 r}$ and we get the desired result.

\section{Direct Method}

In this section, using direct method, we prove the Hyers-Ulam stability of functional equation $(1.8)$ in fuzzy Banach spaces. Throughout this section, we assume that $X$ is a linear space, $(Y, N)$ is a fuzzy Banach space, and $\left(Z, N^{\prime}\right)$ is a fuzzy normed space. Moreover, we assume that $N(x, \cdot)$ is a left continuous function on $\mathbb{R}$.

Theorem 3.1. Assume that a mapping $f: X \rightarrow Y$ is an odd mapping with $f(0)=0$ satisfying the inequality

$$
\begin{aligned}
& N\left(f(k(x+y))+f(k(x-y))-f(k x+y)-f(k x-y)-\frac{2(k+1)}{k} f(k y)+2(k+1) f(y), t\right) \\
& \quad \geq N^{\prime}(\varphi(x, y), t)
\end{aligned}
$$

for all $x, y \in X, t>0$, and $\varphi: X^{2} \rightarrow Z$ is a mapping for which there is a constant $r \in \mathbb{R}$ satisfying $0<|r|<1 /|k|$ such that

$$
N^{\prime}\left(\varphi\left(\frac{x}{k}, \frac{y}{k}\right), t\right) \geq N^{\prime}\left(\varphi(x, y), \frac{t}{|r|}\right)
$$


for all $x, y \in X$ and all $t>0$. Then there exists a unique additive mapping $A: X \rightarrow Y$ satisfying (1.8) and the inequality

$$
N(f(x)-A(x), t) \geq N^{\prime}\left(\varphi(0, x), \frac{|2 k+2|(1-|k r|) t}{|r|}\right)
$$

for all $x \in X$ and all $t>0$.

Proof. It follows from (3.2) that

$$
N^{\prime}\left(\varphi\left(\frac{x}{k^{j}}, \frac{y}{k^{j}}\right), t\right) \geq N^{\prime}\left(\varphi(x, y), \frac{t}{|r|^{j}}\right)
$$

for all $x, y \in X$ and all $t>0$. Putting $x=0$ in (3.1) and then replacing $y$ by $x / k$, we get

$$
N\left(k f\left(\frac{x}{k}\right)-f(x), \frac{|k| t}{|2 k+2|}\right) \geq N^{\prime}\left(\varphi\left(0, \frac{x}{k}\right), t\right)
$$

for all $x \in X$ and all $t>0$. Replacing $x$ by $x / k^{j}$ in (3.5), we have

$$
N\left(k^{j+1} f\left(\frac{x}{k^{j+1}}\right)-k^{j} f\left(\frac{x}{k^{j}}\right), \frac{|k|^{j+1} t}{|2 k+2|}\right) \geq N^{\prime}\left(\varphi\left(0, \frac{x}{k^{j+1}}\right), t\right) \geq N^{\prime}\left(\varphi(0, x), \frac{t}{|r|^{j+1}}\right),
$$

for all $x \in X$, all $t>0$, and all integer $j \geq 0$. So

$$
\begin{aligned}
& N\left(f(x)-k^{n} f\left(\frac{x}{k^{n}}\right), \sum_{j=0}^{n-1} \frac{|k|^{j+1}|r|^{j+1} t}{|2 k+2|}\right) \\
& \quad=N\left(\sum_{j=0}^{n-1} k^{j+1} f\left(\frac{x}{k^{j+1}}\right)-k^{j} f\left(\frac{x}{k^{j}}\right), \sum_{j=0}^{n-1} \frac{|k|^{j+1}|r|^{j+1} t}{|2 k+2|}\right) \\
& \quad \geq \min _{0 \leq j \leq n-1}\left\{N\left(k^{j+1} f\left(\frac{x}{k^{j+1}}\right)-k^{j} f\left(\frac{x}{k^{j}}\right), \frac{|k|^{j+1}|r|^{j+1} t}{|2 k+2|}\right)\right\} \\
& \geq \min _{0 \leq j \leq n-1}\left\{N^{\prime}(\varphi(0, x), t)\right\} \\
& =N^{\prime}(\varphi(0, x), t),
\end{aligned}
$$

which yields

$$
N\left(k^{n+p} f\left(\frac{x}{k^{n+p}}\right)-k^{p} f\left(\frac{x}{k^{p}}\right), \sum_{j=0}^{n-1} \frac{|k|^{j+p+1}|r|^{j+1} t}{|2 k+2|}\right) \geq N^{\prime}\left(\varphi\left(0, \frac{x}{2^{p}}\right), t\right) \geq N^{\prime}\left(\varphi(0, x), \frac{t}{|r|^{p}}\right)
$$


for all $x \in X, t>0$, and all integers $n>0, p \geq 0$. So

$$
N\left(k^{n+p} f\left(\frac{x}{k^{n+p}}\right)-k^{p} f\left(\frac{x}{k^{p}}\right), \sum_{j=0}^{n-1} \frac{|k|^{j+p+1}|r|^{j+p+1} t}{|2 k+2|}\right) \geq N^{\prime}(\varphi(0, x), t),
$$

for all $x \in X, t>0$, and any integers $n>0, p \geq 0$. Hence one can obtain

$$
N\left(k^{n+p} f\left(\frac{x}{k^{n+p}}\right)-k^{p} f\left(\frac{x}{k^{p}}\right), t\right) \geq N^{\prime}\left(\varphi(0, x), \frac{t}{\sum_{j=0}^{n-1}\left(|k|^{j+p+1}|r|^{j+p+1} /|2 k+2|\right)}\right)
$$

for all $x \in X, t>0$, and any integers $n>0, p \geq 0$. Since the series $\sum_{j=0}^{+\infty} k^{j}|r|^{j}$ is a convergent series, we see by taking the limit $p \rightarrow \infty$ in the last inequality that the sequence $\left\{k^{n} f\left(x / k^{n}\right)\right\}$ is a Cauchy sequence in the fuzzy Banach space $(Y, N)$ and so it converges in $Y$. Therefore a mapping $A: X \rightarrow Y$ defined by $A(x):=N-\lim _{n \rightarrow \infty} k^{n} f\left(x / k^{n}\right)$ is well defined for all $x \in X$. This means that

$$
\lim _{n \rightarrow \infty} N\left(A(x)-k^{n} f\left(\frac{x}{k^{n}}\right), t\right)=1
$$

for all $x \in X$ and all $t>0$. In addition, it follows from (3.10) that

$$
N\left(f(x)-k^{n} f\left(\frac{x}{k^{n}}\right), t\right) \geq N^{\prime}\left(\varphi(0, x), \frac{t}{\sum_{j=0}^{n-1}\left(|k|^{j+1}|r|^{j+1} /|2 k+2|\right)}\right),
$$

for all $x \in X$ and all $t>0$. So

$$
\begin{aligned}
N(f(x)-A(x), t) & \geq \min \left\{N\left(f(x)-k^{n} f\left(\frac{x}{k^{n}}\right),(1-\epsilon) t\right), N\left(A(x)-k^{n} f\left(\frac{x}{k^{n}}\right), \epsilon t\right)\right\} \\
& \geq N^{\prime}\left(\varphi(0, x), \frac{\epsilon t}{\sum_{j=0}^{n-1}\left(|k|^{j+1}|r|^{j+1} /|2 k+2|\right)}\right) \\
& \geq N^{\prime}\left(\varphi(0, x), \frac{|2 k+2|(1-|k||r|) \epsilon t}{|k r|}\right),
\end{aligned}
$$

for sufficiently large $n$ and for all $x \in X, t>0$, and $\epsilon$ with $0<\epsilon<1$. Since $\varepsilon$ is arbitrary and $N^{\prime}$ is left continuous, we obtain

$$
N(f(x)-A(x), t) \geq N^{\prime}\left(\varphi(0, x), \frac{|2 k+2|(1-|k||r|) t}{|k r|}\right)
$$


for all $x \in X$ and $t>0$. It follows from (3.1) that

$$
\begin{aligned}
N( & \frac{f\left(k^{n}(x+k y)\right)}{k^{n}}+\frac{f\left(k^{n}(x-k y)\right)}{k^{n}}-\frac{f\left(k^{n}(x+y)\right)}{k^{n}}-\frac{f\left(k^{n}(x-y)\right)}{k^{n}} \\
& \left.-\frac{2(k+1)}{k} \frac{f\left(k^{n+1} y\right)}{k^{n}}+2(k+1) \frac{f\left(k^{n} y\right)}{k^{n}}, t\right) \\
& \geq N^{\prime}\left(\varphi\left(k^{n} x, k^{n} y\right), \frac{t}{|k|^{n}}\right) \geq N^{\prime}\left(\varphi(x, y), \frac{t}{|r|^{n}|k|^{n}}\right) \rightarrow 1 \text { as } n \longrightarrow+\infty,
\end{aligned}
$$

for all $x, y \in X$ and all $t>0$. Therefore, we obtain in view of (3.11)

$$
\begin{aligned}
& N\left(A(k(x+y))+A(k(x-y))-A(k x+y)-A(k x-y)-\frac{2(k+1)}{k} A(k y)\right. \\
& +2(k+1) A(y), t) \\
& \geq \min \left\{N \left(A(k(x+y))+A(k(x-y))-A(k x+y)-A(k x-y)-\frac{2(k+1)}{k} A(k y)\right.\right. \\
& +2(k+1) A(y)-\frac{f\left(k^{n}(x+k y)\right)}{k^{n}}+\frac{f\left(k^{n}(x-k y)\right)}{k^{n}}-\frac{f\left(k^{n}(x+y)\right)}{k^{n}} \\
& \left.-\frac{f\left(k^{n}(x-y)\right)}{k^{n}}-\frac{2(k+1)}{k} \frac{f\left(k^{n+1} y\right)}{k^{n}}+2(k+1) \frac{f\left(k^{n} y\right)}{k^{n}}, \frac{t}{2}\right), \\
& N\left(\frac{f\left(k^{n}(x+k y)\right)}{k^{n}}+\frac{f\left(k^{n}(x-k y)\right)}{k^{n}}-\frac{f\left(k^{n}(x+y)\right)}{k^{n}}-\frac{f\left(k^{n}(x-y)\right)}{k^{n}}\right. \\
& \left.\left.-\frac{2(k+1)}{k} \frac{f\left(k^{n+1} y\right)}{k^{n}}+2(k+1) \frac{f\left(k^{n} y\right)}{k^{n}}, \frac{t}{2}\right)\right\} \\
& =N\left(\frac{f\left(k^{n}(x+k y)\right)}{k^{n}}+\frac{f\left(k^{n}(x-k y)\right)}{k^{n}}-\frac{f\left(k^{n}(x+y)\right)}{k^{n}}-\frac{f\left(k^{n}(x-y)\right)}{k^{n}}\right. \\
& \left.-\frac{2(k+1)}{k} \frac{f\left(k^{n+1} y\right)}{k^{n}}+2(k+1) \frac{f\left(k^{n} y\right)}{k^{n}}, \frac{t}{2}\right) \quad(\text { for sufficiently large } n) \\
& \geq N^{\prime}\left(\varphi(x, y), \frac{t}{2|k|^{n}|r|^{n}}\right) \longrightarrow 1 \text { as } n \longrightarrow+\infty \text {, }
\end{aligned}
$$

for all $x, y \in X$ and all $t>0$, which implies that

$$
A(k(x+y))+A(k(x-y))=A(k x+y)+A(k x-y)+\frac{2(k+1)}{k} A(k y)-2(k+1) A(y)
$$

Hence the mapping $A: X \rightarrow Y$ is additive, as desired. 
To prove the uniqueness, let there be another mapping $L: X \rightarrow Y$ which satisfies the inequality (3.3). Since $L\left(k^{n} x\right)=k^{n} L(x)$ for all $x \in X$, we have

$$
\begin{aligned}
N(A(x)-L(x), t) & =N\left(k^{n} A\left(\frac{x}{k^{n}}\right)-k^{n} L\left(\frac{x}{k^{n}}\right), t\right) \\
& \geq \min \left\{N\left(k^{n} A\left(\frac{x}{k^{n}}\right)-k^{n} f\left(\frac{x}{k^{n}}\right), \frac{t}{2}\right), N\left(k^{n} f\left(\frac{x}{k^{n}}\right)-k^{n} L\left(\frac{x}{k^{n}}\right), \frac{t}{2}\right)\right\} \\
& \geq N^{\prime}\left(\varphi\left(0, \frac{x}{k^{n}}\right), \frac{|2 k+2|(1-|k||r|) t}{2|k|^{n+1}|r|}\right) \\
& \geq N^{\prime}\left(\varphi(0, x), \frac{|2 k+2|(1-|k||r|) t}{2|k|^{n+1}|r|^{n+1}}\right) \longrightarrow 1 \text { as } n \longrightarrow \infty,
\end{aligned}
$$

for all $t>0$. Therefore $A(x)=L(x)$ for all $x \in X$. This completes the proof.

Corollary 3.2. Let $X$ be a normed space and let $\left(\mathbb{R}, N^{\prime}\right)$ be a fuzzy Banach space. Assume that there exist real numbers $\theta \geq 0$ and $p>1$ such that an odd mapping $f: X \rightarrow Y$ with $f(0)=0$ satisfies the following inequality:

$$
\begin{aligned}
& N\left(f(k(x+y))+f(k(x-y))-f(k x+y)-f(k x-y)-\frac{2(k+1)}{k} f(k y)+2(k+1) f(y), t\right) \\
& \quad \geq N^{\prime}\left(\theta\left(\|x\|^{p}+\|y\|^{p}\right), t\right),
\end{aligned}
$$

for all $x, y \in X$ and $t>0$. Then there is a unique additive mapping $A: X \rightarrow Y$ satisfying (1.8) and the inequality

$$
N(f(x)-A(x), t) \geq N^{\prime}\left(\frac{\theta\|x\|^{p}}{|2 k+2|},\left(\frac{|k|^{p}-|k|}{|k|}\right) t\right) .
$$

Proof. Let $\varphi(x, y):=\theta\left(\|x\|^{p}+\|y\|^{p}\right)$ and $|r|=|k|^{-p}$. Applying Theorem 3.1, we get desired results.

Theorem 3.3. Let $f: X \rightarrow Y$ be an odd mapping with $f(0)=0$ satisfying the inequality (3.1) and let $\varphi: X^{2} \rightarrow Z$ be a mapping for which there exists a constant $r \in \mathbb{R}$ satisfying $0<|r|<|k|$ such that

$$
N^{\prime}(\varphi(x, y),|r| t) \geq N^{\prime}\left(\varphi\left(\frac{x}{k}, \frac{y}{k}\right), t\right)
$$


for all $x, y \in X$ and all $t>0$. Then there exists a unique additive mapping $A: X \rightarrow Y$ satisfying (1.8) and the following inequality:

$$
N(f(x)-A(x), t) \geq N^{\prime}\left(\varphi(0, x), \frac{|2 k+2|(|k|-|r|) t}{|k|}\right),
$$

for all $x \in X$ and all $t>0$.

Proof. It follows from (3.5) that

$$
N\left(\frac{f(k x)}{k}-f(x), \frac{t}{|2 k+2|}\right) \geq N^{\prime}(\varphi(0, x), t),
$$

for all $x \in X$ and all $t>0$. Replacing $x$ by $k^{n} x$ in (3.41), we obtain

$$
N\left(\frac{f\left(k^{n+1} x\right)}{k^{n+1}}-\frac{f\left(k^{n} x\right)}{k^{n}}, \frac{t}{|2 k+2| k^{n}}\right) \geq N^{\prime}\left(\varphi\left(0, k^{n} x\right), t\right) \geq N^{\prime}\left(\varphi(0, x), \frac{t}{|r|^{n}}\right) .
$$

So

$$
N\left(\frac{f\left(k^{n+1} x\right)}{k^{n+1}}-\frac{f\left(k^{n} x\right)}{k^{n}}, \frac{|r|^{n} t}{|2 k+2||k|^{n}}\right) \geq N^{\prime}(\varphi(0, x), t),
$$

for all $x \in X$ and all $t>0$. Proceeding as in the proof of Theorem 3.1, we obtain that

$$
N\left(f(x)-\frac{f\left(k^{n} x\right)}{k^{n}}, \sum_{j=0}^{n-1} \frac{|r|^{j} t}{|2 k+2||k|^{j}}\right) \geq N^{\prime}(\varphi(0, x), t)
$$

for all $x \in X$, all $t>0$, and any integer $n>0$. So

$$
N\left(f(x)-\frac{f\left(k^{n} x\right)}{k^{n}}, t\right) \geq N^{\prime}\left(\varphi(0, x), \frac{t}{\sum_{j=0}^{n-1}\left(|r|^{j} /|2 k+2||k|^{j}\right)}\right) .
$$

The rest of the proof is similar to the proof of Theorem 3.1.

Corollary 3.4. Let $X$ be a normed space and let $\left(\mathbb{R}, N^{\prime}\right)$ be a fuzzy Banach space. Assume that there exist real numbers $\theta \geq 0$ and $0<p<1$ such that an odd mapping $f: X \rightarrow Y$ with $f(0)=0$ satisfies (3.19). Then there exists a unique additive mapping $A: X \rightarrow Y$ satisfying (1.8) and the inequality

$$
N(f(x)-A(x), t) \geq N^{\prime}\left(\varphi(0, x), \frac{|2 k+2|\left(|k|-|k|^{p}\right) t}{|k|}\right) .
$$


Proof. Let $\varphi(x, y):=\theta\left(\|x\|^{p}+\|y\|^{p}\right)$ and $|r|=|k|^{p}$. Applying Theorem 3.3, we get the desired results.

Theorem 3.5. Let $f: X \rightarrow Y$ be an even mapping with $f(0)=0$ satisfying the inequality (3.1) and let $\varphi: X^{2} \rightarrow Z$ be a mapping for which there exists a constant $r \in \mathbb{R}$ such that $0<|r|<1 / k^{2}$ and that

$$
N^{\prime}\left(\varphi\left(\frac{x}{k}, \frac{y}{k}\right), t\right) \geq N^{\prime}\left(\varphi(x, y), \frac{t}{|r|}\right)
$$

for all $x, y \in X$ and all $t>0$. Then there exists a unique quadratic mapping $Q: X \rightarrow Y$ satisfying (1.8) and the inequality

$$
N(f(x)-Q(x), t) \geq N^{\prime}\left(\varphi(0, x), \frac{2\left(1-\left|k^{2} r\right|\right) t}{|k r|}\right),
$$

for all $x \in X$ and all $t>0$.

Proof. Replacing $x$ by $k x$ in (3.1), we get

$$
\begin{aligned}
& N\left(f(k(x+y))+f(k(x-y))-f(k x+y)-f(k x-y)-\frac{2(k+1)}{k} f(k y)+2(k+1) f(y), t\right) \\
& \quad \geq N^{\prime}(\varphi(k x, y), t),
\end{aligned}
$$

for all $x, y \in X$ and all $t>0$. Putting $x=0$ and replacing $y$ by $x$ in (3.31), we have

$$
N\left(\frac{f(k x)}{k^{2}}-f(x), \frac{t}{|2 k|}\right) \geq N^{\prime}(\varphi(0, x), t),
$$

for all $x \in X$ and all $t>0$. Replacing $x$ by $x / k$ in (3.32), we find

$$
N\left(k^{2} f\left(\frac{x}{k}\right)-f(x), \frac{|k| t}{2}\right) \geq N^{\prime}\left(\varphi\left(0, \frac{x}{k}\right), t\right),
$$

for all $x \in X$ and all $t>0$. Also, replacing $x$ by $x / k^{n}$ in (3.33), we obtain

$$
N\left(k^{2 n+2} f\left(\frac{x}{k^{n}}\right)-k^{2 n} f\left(\frac{x}{k^{n}}\right), \frac{|k|^{2 n+1} t}{2}\right) \geq N^{\prime}\left(\varphi\left(0, \frac{x}{k^{n+1}}\right), t\right) \geq N^{\prime}\left(\varphi(0, x), \frac{t}{|r|^{n+1}}\right) .
$$

So

$$
N\left(k^{2 n+2} f\left(\frac{x}{k^{n}}\right)-k^{2 n} f\left(\frac{x}{k^{n}}\right), \frac{|k|^{2 n+1}|r|^{n+1} t}{2}\right) \geq N^{\prime}(\varphi(0, x), t)
$$


for all $x \in X$ and all $t>0$. Proceeding as in the proof of Theorem 3.1, we obtain that

$$
N\left(f(x)-k^{2 n} f\left(\frac{x}{k^{n}}\right), \sum_{j=0}^{n-1} \frac{|k|^{2 j+1}|r|^{j+1} t}{2}\right) \geq N^{\prime}(\varphi(0, x), t),
$$

for all $x \in X$, all $t>0$, and any integer $n>0$. So

$$
N\left(f(x)-k^{2 n} f\left(\frac{x}{k^{n}}\right), t\right) \geq N^{\prime}\left(\varphi(0, x), \frac{t}{\sum_{j=0}^{n-1}\left(|k|^{2 j+1}|r|^{j+1} t / 2\right)}\right) .
$$

The rest of the proof is similar to the proof of Theorem 3.1.

Corollary 3.6. Let $X$ be a normed space and let $\left(\mathbb{R}, N^{\prime}\right)$ be a fuzzy Banach space. Assume that there exist real numbers $\theta \geq 0$ and $p>1$ such that an even mapping $f: X \rightarrow Y$ with $f(0)=0$ satisfies the inequality (3.19). Then there exists a unique quadratic mapping $Q: X \rightarrow Y$ satisfying (1.8) and the inequality

$$
N(f(x)-Q(x), t) \geq N^{\prime}\left(\theta\|x\|^{p}, \frac{2\left(k^{2 p}-k^{2}\right) t}{|k|}\right)
$$

Proof. Let $\varphi(x, y):=\theta\left(\|x\|^{p}+\|y\|^{p}\right)$ and $|r|=|k|^{-2 p}$. Applying Theorem 3.5, we get the desired results.

Theorem 3.7. Assume that an even mapping $f: X \rightarrow Y$ with $f(0)=0$ satisfies the inequality (3.1) and $\varphi: X^{2} \rightarrow Z$ is a mapping for which there is a constant $r \in \mathbb{R}$ satisfying $0<|r|<k^{2}$ such that

$$
N^{\prime}(\varphi(x, y),|r| t) \geq N^{\prime}\left(\varphi\left(\frac{x}{k}, \frac{y}{k}\right), t\right)
$$

for all $x, y \in X$ and all $t>0$. Then there exists a unique quadratic mapping $Q: X \rightarrow Y$ satisfying (1.8) and the following inequality

$$
N(f(x)-Q(x), t) \geq N^{\prime}\left(\varphi(0, x), \frac{2\left(k^{2}-|r|\right) t}{|k|}\right)
$$

for all $x \in X$ and all $t>0$.

Proof. It follows from (3.32) that

$$
N\left(\frac{f(k x)}{k^{2}}-f(x), \frac{t}{|2 k|}\right) \geq N^{\prime}(\varphi(0, x), t),
$$


for all $x \in X$ and all $t>0$. Replacing $x$ by $k^{n} x$ in (3.41), we obtain

$$
N\left(\frac{f\left(k^{n+1} x\right)}{k^{2 n+2}}-\frac{f\left(k^{n} x\right)}{k^{2 n}}, \frac{t}{2|k|^{2 n+1}}\right) \geq N^{\prime}\left(\varphi\left(0, k^{n} x\right), t\right) \geq N^{\prime}\left(\varphi(0, x), \frac{t}{|r|^{n}}\right)
$$

for all $x \in X$ and all $t>0$. So

$$
N\left(\frac{f\left(k^{n+1} x\right)}{k^{2 n+2}}-\frac{f\left(k^{n} x\right)}{k^{2 n}}, \frac{|r|^{n} t}{2|k|^{2 n+1}}\right) \geq N^{\prime}(\varphi(0, x), t),
$$

for all $x \in X$ and all $t>0$. So

$$
N\left(f(x)-\frac{f\left(k^{n} x\right)}{k^{2 n}}, t\right) \geq N^{\prime}\left(\varphi(0, x), \frac{t}{\sum_{j=0}^{n-1}\left(|r|^{j} t / 2|k|^{2 j+1}\right)}\right) .
$$

The rest of the proof is similar to the proof of Theorem 3.1.

Corollary 3.8. Let $X$ be a normed space and let $\left(\mathbb{R}, N^{\prime}\right)$ be a fuzzy Banach space. Assume that there exist real numbers $\theta \geq 0$ and $0<p<1$ such that an even mapping $f: X \rightarrow Y$ with $f(0)=0$ satisfies (3.19). Then there is a unique quadratic mapping $Q: X \rightarrow Y$ satisfying (1.8) and the inequality

$$
N(f(x)-Q(x), t) \geq N^{\prime}\left(\varphi(0, x), \frac{2\left(k^{2}-k^{2 p}\right) t}{|k|}\right)
$$

for all $x \in X$, all $t>0$.

Proof. Let $\varphi(x, y):=\theta\left(\|x\|^{p}+\|y\|^{p}\right)$ and $|r|=k^{2 p}$. Applying Theorem 3.7, we get the desired results.

\section{Acknowledgment}

The fifth author of this work was partially supported by Basic Science Research Program through the National Research Foundation of Korea (NRF) funded by the Ministry of Education, Science and Technology (Grant no. 2010-0010243).

\section{References}

[1] S. M. Ulam, Problems in Modern Mathematics, John Wiley \& Sons, Inc, New York, NY, USA, 1964.

[2] D. H. Hyers, "On the stability of the linear functional equation," Proceedings of the National Academy of Sciences of the United States of America, vol. 27, pp. 222-224, 1941.

[3] T. M. Rassias, "On the stability of the linear mapping in Banach spaces," Proceedings of the American Mathematical Society, vol. 72, no. 2, pp. 297-300, 1978.

[4] Z. Gajda, "On stability of additive mappings," International Journal of Mathematics and Mathematical Sciences, vol. 14, no. 3, pp. 431-434, 1991. 
[5] S. Abbaszadeh, "Intuitionistic fuzzy stability of a quadratic and quartic functional equation," International Journal of Nonlinear Analysis and Applications, vol. 1, no. 2, pp. 100-124, 2010.

[6] J. Aczél and J. Dhombres, Functional Equations in Several Variables, vol. 31, Cambridge University Press, Cambridge, UK, 1989.

[7] T. Aoki, "On the stability of the linear transformation in Banach spaces," Journal of the Mathematical Society of Japan, vol. 2, pp. 64-66, 1950.

[8] D. G. Bourgin, "Classes of transformations and bordering transformations," Bulletin of the American Mathematical Society, vol. 57, pp. 223-237, 1951.

[9] M. B. Savadkouhi, M. E. Gordji, J. M. Rassias, and N. Ghobadipour, "Approximate ternary Jordan derivations on Banach ternary algebras," Journal of Mathematical Physics, vol. 50, no. 4, article 042303, p. 9, 2009.

[10] P. Găvruţa, "A generalization of the Hyers-Ulam-Rassias stability of approximately additive mappings," Journal of Mathematical Analysis and Applications, vol. 184, no. 3, pp. 431-436, 1994.

[11] P. Găvruța and L. Găvruţa, “A new method for the generalized Hyers-Ulam-Rassias stability,” Journal of Mathematical Analysis and Applications, vol. 1, no. 2, pp. 11-18, 2010.

[12] D. H. Hyers, G. Isac, and T. M. Rassias, Stability of Functional Equations in Several Variables, Progress in Nonlinear Differential Equations and their Applications, 34, Birkhäuser Boston Inc., Boston, Mass, USA, 1998.

[13] G. Isac and T. M. Rassias, "On the Hyers-Ulam stability of $\psi$-additive mappings," Journal of Approximation Theory, vol. 72, no. 2, pp. 131-137, 1993.

[14] C. Park and M. E. Gordji, "Comment on "Approximate ternary Jordan derivations on Banach ternary algebras" [B. Savadkouhi Journal of Mathematical Physics vol. 50, article 042303, 2009]," Journal of Mathematical Physics, vol. 51, no. 4, 2010.

[15] C. Park and A. Najati, "Generalized additive functional inequalities in Banach algebras," International Journal of Nonlinear Analysis and Applications, vol. 1, no. 2, pp. 54-62, 2010.

[16] C. Park and T. M. Rassias, "Isomorphisms in unital $C^{*}$-algebras," International Journal of Nonlinear Analysis and Applications, vol. 1, no. 2, pp. 1-10, 2010.

[17] T. M. Rassias, "On the stability of functional equations and a problem of Ulam," Acta Applicandae Mathematicae, vol. 62, no. 1, pp. 23-130, 2000.

[18] P. Kannappan, "Quadratic functional equation and inner product spaces," Results in Mathematics, vol. 27, no. 3-4, pp. 368-372, 1995.

[19] F. Skof, "Local properties and approximation of operators," Rendiconti del Seminario Matematico e Fisico di Milano, vol. 53, pp. 113-129, 1983.

[20] P. W. Cholewa, "Remarks on the stability of functional equations," Aequationes Mathematicae, vol. 27, no. 1-2, pp. 76-86, 1984.

[21] S. Czerwik, "On the stability of the quadratic mapping in normed spaces," Abhandlungen aus dem Mathematischen Seminar der Universität Hamburg, vol. 62, pp. 59-64, 1992.

[22] A. Grabiec, "The generalized Hyers-Ulam stability of a class of functional equations," Publicationes Mathematicae Debrecen, vol. 48, no. 3-4, pp. 217-235, 1996.

[23] C. Borelli and G. L. Forti, "On a general Hyers-Ulam stability result," International Journal of Mathematics and Mathematical Sciences, vol. 18, no. 2, pp. 229-236, 1995.

[24] C.-G. Park, "Generalized quadratic mappings in several variables," Nonlinear Analysis. Theory, Methods E Applications, vol. 57, no. 5-6, pp. 713-722, 2004.

[25] C.-G. Park, "On the stability of the quadratic mapping in Banach modules," Journal of Mathematical Analysis and Applications, vol. 276, no. 1, pp. 135-144, 2002.

[26] A. Ebadian, A. Najati, and M. Eshaghi Gordji, “On approximate additive-quartic and quadratic-cubic functional equations in two variables on abelian groups," Results in Mathematics, vol. 58, no. 1-2, pp. 39-53, 2010.

[27] M. Eshaghi Gordji, "Stability of a functional equation deriving from quartic and additive functions," Bulletin of the Korean Mathematical Society, vol. 47, no. 3, pp. 491-502, 2010.

[28] M. Eshaghi Gordji, "Stability of an additive-quadratic functional equation of two variables in Fspaces," Journal of Nonlinear Science and its Applications, vol. 2, no. 4, pp. 251-259, 2009.

[29] M. Eshaghi Gordji and M. B. Savadkouhi, "Stability of cubic and quartic functional equations in nonArchimedean spaces," Acta Applicandae Mathematicae, vol. 110, no. 3, pp. 1321-1329, 2010.

[30] M. Eshaghi Gordji and M. B. Savadkouhi, "Stability of a mixed type cubic-quartic functional equation in non-Archimedean spaces," Applied Mathematics Letters, vol. 23, no. 10, pp. 1198-1202, 2010. 
[31] M. Eshaghi Gordji and M. B. Savadkouhi, "Approximation of generalized homomorphisms in quasiBanach algebras," Mathematical Journal of the Ovidius University of Constantza, vol. 17, no. 2, pp. $203-$ 213, 2009.

[32] M. Eshaghi Gordji and M. Bavand Savadkouhi, "On approximate cubic homomorphisms," Advances in Difference Equations, vol. 2009, Article ID 618463, 11 pages, 2009.

[33] M. Eshaghi Gordji, S. Zolfaghari, J. M. Rassias, and M. B. Savadkouhi, "Solution and stability of a mixed type cubic and quartic functional equation in quasi-Banach spaces," Abstract and Applied Analysis, vol. 2009, Article ID 417473, 14 pages, 2009.

[34] M. Eshaghi Gordji and M. B. Savadkouhi, "Stability of mixed type cubic and quartic functional equations in random normed spaces," Journal of Inequalities and Applications, vol. 2009, Article ID 527462, 9 pages, 2009.

[35] M. Eshaghi Gordji, A. Ebadian, and S. Zolfaghari, "Stability of a functional equation deriving from cubic and quartic functions," Abstract and Applied Analysis, vol. 2008, Article ID 801904, 17 pages, 2008.

[36] M. Eshaghi Gordji, S. Kaboli Gharetapeh, J. M. Rassias, and S. Zolfaghari, "Solution and stability of a mixed type additive, quadratic, and cubic functional equation," Advances in Difference Equations, vol. 2009, Article ID 826130, 17 pages, 2009.

[37] M. Eshaghi-Gordji, S. Kaboli-Gharetapeh, C. Park, and S. Zolfaghari, "Stability of an additive-cubicquartic functional equation," Advances in Difference Equations, vol. 2009, Article ID 395693, 20 pages, 2009.

[38] M. Eshaghi Gordji, T. Karimi, and S. Kaboli Gharetapeh, "Approximately n-Jordan homomorphisms on Banach algebras," Journal of Inequalities and Applications, vol. 2009, Article ID 870843, 8 pages, 2009.

[39] M. Eshaghi Gordji and H. Khodaei, "Solution and stability of generalized mixed type cubic, quadratic and additive functional equation in quasi-Banach spaces," Nonlinear Analysis. Theory, Methods $\mathcal{E}$ Applications, vol. 71, no. 11, pp. 5629-5643, 2009.

[40] M. Eshaghi Gordji, H. Khodaei, and R. Khodabakhsh, "General quartic-cubic-quadratic functional equation in non-Archimedean normed spaces," "Politehnica" University of Bucharest Scientific Bulletin. Series A, vol. 72, no. 3, pp. 69-84, 2010.

[41] M. Eshaghi Gordji and A. Najati, "Approximately $J^{*}$-homomorphisms: a fixed point approach," Journal of Geometry and Physics, vol. 60, no. 5, pp. 809-814, 2010.

[42] M. Eshaghi Gordji, S. Zolfaghari, S. Kaboli Gharetapeh, A. Ebadian, and C. Park, "Solution and stability of generalized mixed type additive and quadratic functional equation in non-Archimedean spaces," to appear in Annali dell'Universita di Ferrara.

[43] R. Farokhzad and S. A. R. Hosseinioun, "Perturbations of Jordan higher derivations in Banach ternary algebras: an alternative fixed point approach," International Journal of Nonlinear Analysis and Applications, vol. 1, no. 1, pp. 42-53, 2010.

[44] M. Eshaghi Gordji and H. Khodaei, "On the generalized Hyers-Ulam-Rassias stability of quadratic functional equations," Abstract and Applied Analysis, vol. 2009, Article ID 923476, 11 pages, 2009.

[45] M. Eshaghi Gordji, S. Kaboli Gharetapeh, J. M. Rassias, and S. Zolfaghari, "Solution and stability of a mixed type additive, quadratic, and cubic functional equation," Advances in Difference Equations, vol. 2009, Article ID 826130, 17 pages, 2009.

[46] M. Eshaghi Gordji, S. Kaboli Gharetapeh, E. Rashidi, T. Karimi, and M. Aghaei, “Ternary Jordanderivations in $C^{*}$-ternary algebras," Journal of Computational Analysis and Applications, vol. 12, no. 2, pp. 463-470, 2010.

[47] M. Eshaghi Gordji, J. M. Rassias, and N. Ghobadipour, "Generalized Hyers-Ulam stability of generalized (N,K)-derivations," Abstract and Applied Analysis, vol. 2009, Article ID 437931, 8 pages, 2009.

[48] T. Bag and S. K. Samanta, "Finite dimensional fuzzy normed linear spaces," Journal of Fuzzy Mathematics, vol. 11, no. 3, pp. 687-705, 2003.

[49] T. Bag and S. K. Samanta, "Fuzzy bounded linear operators," Fuzzy Sets and Systems, vol. 151, no. 3, pp. 513-547, 2005.

[50] D. Miheţ and V. Radu, "On the stability of the additive Cauchy functional equation in random normed spaces," Journal of Mathematical Analysis and Applications, vol. 343, no. 1, pp. 567-572, 2008. 


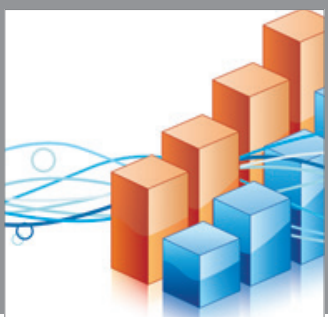

Advances in

Operations Research

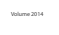

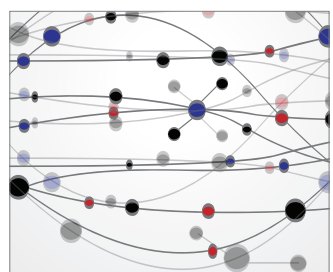

\section{The Scientific} World Journal
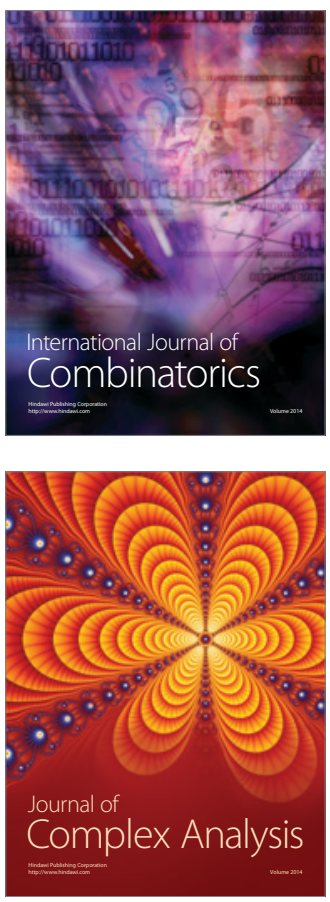

International Journal of

Mathematics and

Mathematical

Sciences
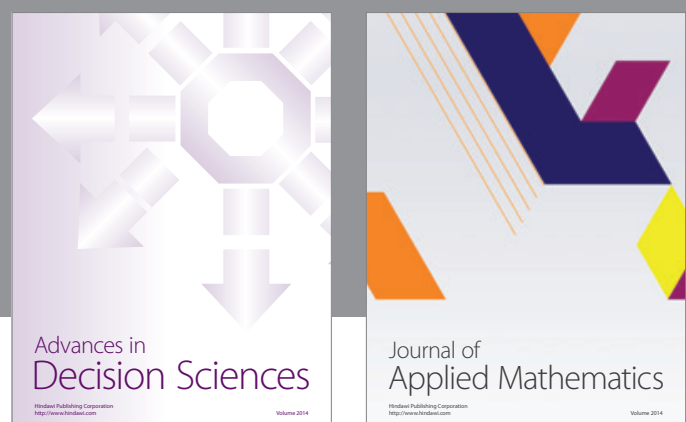

Journal of

Applied Mathematics
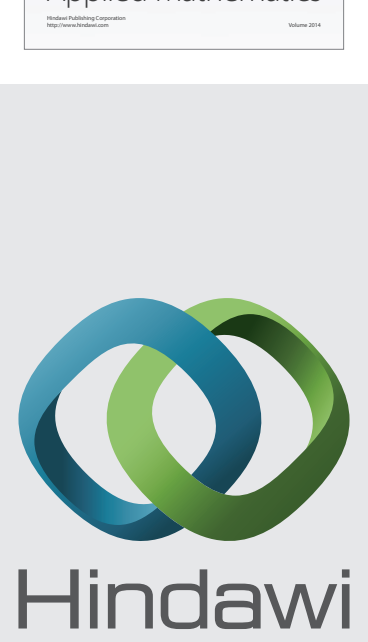

Submit your manuscripts at http://www.hindawi.com
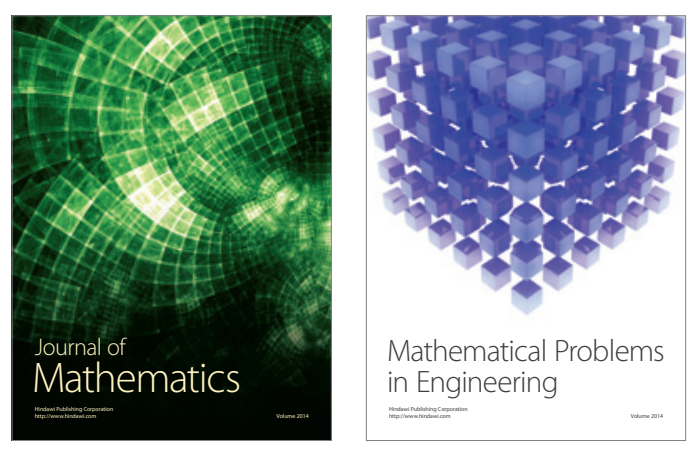

Mathematical Problems in Engineering
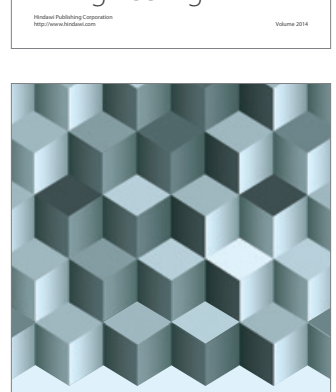

Journal of

Function Spaces
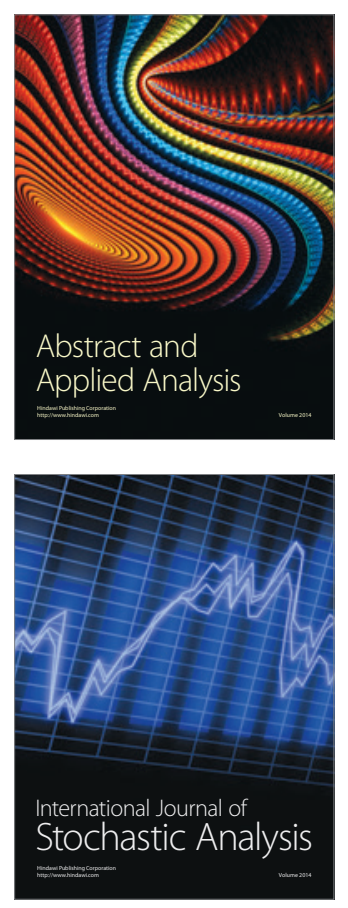

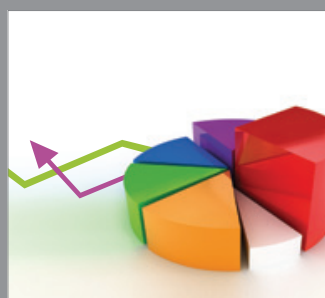

ournal of

Probability and Statistics

Promensencen
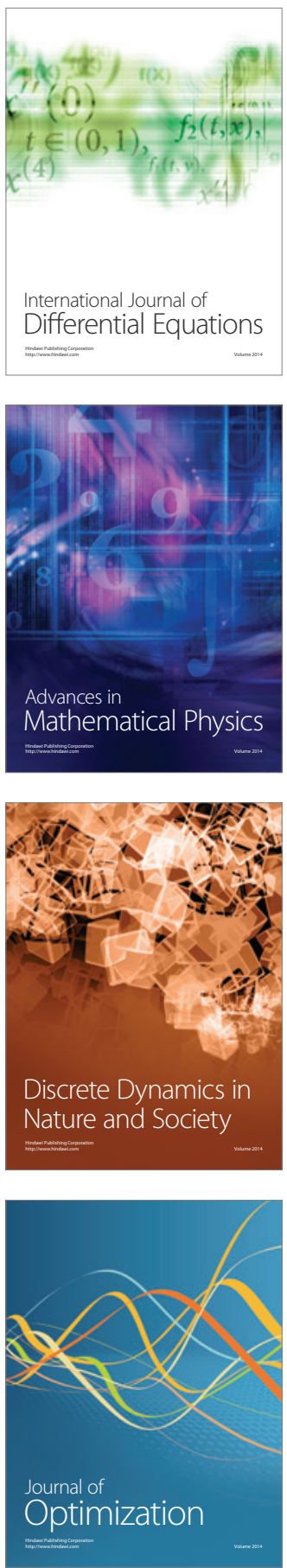